\title{
UNIFORM ASYMPTOTIC STABILITY IN FUNCTIONAL DIFFERENTIAL EQUATIONS
}

\author{
T. A. BURTON
}

ABSTRACT. The classical uniform asymptotic stability result for a system of functional differential equations

$$
x^{\prime}=F\left(t, x_{t}\right)
$$

calls for a Liapunov functional $V(t, \phi)$ satisfying $W(|\phi(0)|)<V(t, \phi)<$ $W_{1}(|\phi(0)|)+W_{2}(|||\phi|||), \quad V_{(1)}^{\prime}<-W_{3}(|\phi(0)|)$, and $\left|f\left(t, x_{t}\right)\right|$ bounded for $\left|\| x_{t}\right|||$ bounded. We show that it is not necessary to require $\left|f\left(t, x_{t}\right)\right|$ bounded. Here, $\||| \cdot||$ is the $L^{2}$-norm.

1. Introduction and notation. We consider a system of functional differential equations

$$
x^{\prime}(t)=F\left(t, x_{t}\right)
$$

and obtain conditions on a Liapunov functional to insure that the zero solution is uniformly asymptotically stable. This work generalizes the classical result in that we do not require the usual bound on $F$ for $x_{t}$ bounded. For reference, our discussion here follows closely that of Yoshizawa [1, pp. 183-192]. Our result is a generalization of his theorem on p. 192.

For $x \in R^{n},|x|$ will be the $\max \left|x_{i}\right|$. For a given $h>0, C$ denotes the space of continuous functions mapping $[-h, 0]$ into $R^{n}$ and for $\phi \in C,\|\phi\|=$ $\sup _{-h \leqslant \theta \leqslant 0}|\phi(\theta)| . C_{H}$ denotes the set of $\phi \in C$ with $\|\phi\| \leqslant H$. If $x$ is a continuous function of $u$ defined on $-h \leqslant u<A, A>0$, and if $t$ is a fixed number satisfying $0 \leqslant t<A$, then $x_{t}$ denotes the restriction of $x$ to the interval $[t-h, t]$ so that $x_{t}$ is an element of $C$ defined by $x_{t}(\theta)=x(t+\theta)$ for $-h \leqslant \theta \leqslant 0$.

In (1), $x^{\prime}(t)$ denotes the right-hand derivative of $x$ at $t$ and $F(t, \phi) \in R^{n}$ is defined on $[0, \infty) \times C_{H}$.

We denote by $x\left(t_{0}, \phi\right)$ a solution of (1) with initial condition $\phi \in C_{H}$ where $x_{t_{0}}\left(t_{0}, \phi\right)=\phi$ and we denote by $x\left(t ; t_{0}, \phi\right)$ the value of $x\left(t_{0}, \phi\right)$ at $t$.

It is assumed that $F(t, \phi)$ is continuous on $[0, \infty) \times C_{H}$ so that a solution will exist for each continuous initial condition. For continuation of solutions, we suppose that $F$ takes closed bounded sets of $[0, \infty) \times C_{H}$ into closed bounded sets of $R^{n}$.

Received by the editors June 30, 1977.

AMS (MOS) subject classifications (1970). Primary 34K20.

Key words and phrases. Functional differential equations, Liapunov functionals, uniform asymptotic stability.

() American Mathematical Society 1978 
Let $V(t, \phi)$ be a continuous functional defined for $t \geqslant 0, \phi \in C_{H}$. The upper right-hand derivative of $V$ along solutions of (1) is defined to be

$$
V_{(1)}^{\prime}\left(t, x_{t}\left(t_{0}, \phi\right)\right)=\varlimsup_{\delta \rightarrow 0^{+}}\left\{V\left(t+\delta, x_{t+\delta}\left(t_{0}, \phi\right)\right)-V\left(t, x_{t}\left(t_{0}, \phi\right)\right)\right\} / \delta .
$$

If $V$ satisfies a Lipschitz condition in the second argument, then this limit is uniquely determined.

We suppose that $F(t, 0)=0$, and we say that the zero solution is uniformly stable if for each $\varepsilon>0$ there exists $\delta>0$ so that $t_{0} \geqslant 0, t \geqslant t_{0}$, and $\|\phi\|<\delta$ imply $\left|x\left(t ; t_{0}, \phi\right)\right|<\varepsilon$. If, in addition, there exists $\delta>0$ such that for any $\varepsilon>0$ there exists $T>0$ such that $t_{0} \geqslant 0, t \geqslant t_{0}+T$, and $\|\phi\|<\delta$ imply $\left|x\left(t ; t_{0}, \phi\right)\right|<\varepsilon$, then $x=0$ is uniformly asymptotically stable (UAS).

If $\phi \in C_{H}$, then

$$
\|\phi\|=\left[\sum_{i=1}^{n} \int_{-h}^{0} \phi_{i}^{2}(s) d s\right]^{1 / 2}
$$

where $\phi_{i}$ is the $i$ th component of $\phi$. Finally, $W, W_{1}, W_{2}$, and $W_{3}$ will denote continuous functions from $[0, \infty)$ into $[0, \infty)$ with $W_{i}(0)=0, W_{i}(r)>0$ if $r>0$, and $W_{i}$ nondecreasing.

2. Uniform asymptotic stability. The following lemma is used in the proof of our result.

LEMMA. Let $\left\{x_{n}\right\}$ be a sequence of continuous functions having continuous derivatives, $x_{n}:[0,1] \rightarrow[0,1]$. Let $g:[0, \infty) \rightarrow[0, \infty)$ be continuous, $g(0)=0$, $g(r)>0$ if $r>0$, and let $g$ be nondecreasing. If there exists $\alpha>0$ with $\int_{0}^{1} x_{n}(t) d t \geqslant \alpha$ for all $n$, then there exists $\beta>0$ with $\int_{0}^{1} g\left(x_{n}(t)\right) d t \geqslant \beta$ for all $n$.

Proof. Let $M_{k}=\left\{t \mid x_{k}(t) \geqslant \alpha / 2\right\}, M_{k}^{c}$ be the complement of $M_{k}$ on [0, 1], and let $m_{k}$ be the measure of $M_{k}$. Then $m_{k} \geqslant \alpha / 2$. To see this, if $m_{k}<\alpha / 2$, then

$$
\alpha \leqslant \int_{0}^{1} x_{k}(t) d t=\int_{M_{k}} x_{k}(t) d t+\int_{M_{k}^{c}} x_{k}(t) d t<\frac{\alpha}{2}+\frac{\alpha}{2},
$$

a contradiction. (The first $\alpha / 2$ follows from $m_{k}<\alpha / 2$ and $x_{k}(t) \leqslant 1$. The second $\alpha / 2$ follows from meas $M_{k}^{c} \leqslant 1$ and $x_{k}(t) \leqslant \alpha / 2$.) Hence, for any $k$, we have

$$
\begin{aligned}
\int_{0}^{1} g\left(x_{k}(t)\right) d t & \geqslant \int_{M_{k}} g\left(x_{k}(t)\right) d t \geqslant \int_{M_{k}} g(\alpha / 2) d t \\
& =m_{k} g(\alpha / 2) \geqslant g(\alpha / 2) \alpha / 2 \stackrel{\text { def }}{=} \beta .
\end{aligned}
$$

This completes the proof.

The following result is known when $|F(t, \phi)| \leqslant L$ for $0 \leqslant t<\infty$ and $\|\phi\|<H$.

THEOREM 1. Suppose there is a continuous functional $V(t, \phi)$, locally 
Lipschitz in $\phi$, defined on $0 \leqslant t<\infty,\|\phi\|<H$ which satisfies:

(i) $W(|\phi(0)|) \leqslant V(t, \phi) \leqslant W_{1}(|\phi(0)|)+W_{2}(|||\phi|||)$ and

(ii) $V_{(1)}^{\prime}(t, \phi) \leqslant-W_{3}(|\phi(0)|)$.

Then the zero solution of (1) is uniformly asymptotically stable.

Proof. For a given $\varepsilon>0(\varepsilon<H)$, choose $\delta>0$ so that $W_{1}(\delta)+$ $W_{2}\left(\left[\delta^{2} n h\right]^{1 / 2}\right)<W(\varepsilon)$. Then for an initial function $\phi \in C_{H}$ satisfying $\|\phi\|<$ $\delta$ we have $V_{(1)}^{\prime}\left(t, x_{t}\left(t_{0}, \phi\right)\right) \leqslant 0$ and so if $x(t)=x\left(t ; t_{0}, \phi\right)$, then

$$
\begin{aligned}
W(|x(t)|) & \leqslant V\left(t, x_{t}\left(t_{0}, \phi\right)\right) \leqslant V\left(t_{0}, \phi\right) \\
& \leqslant W_{1}(|\phi(0)|)+W_{2}(|||\phi|||) \\
& <W_{1}(\delta)+W_{2}\left(\left[\delta^{2} n h\right]^{1 / 2}\right)<W(\varepsilon)
\end{aligned}
$$

so that $|x(t)|<\varepsilon$ for $t \geqslant t_{0}$ by the monotonicity of $W$. Thus, $x=0$ is uniformly stable.

Next, given $H>0$, find the $\delta$ of uniform stability for $\min [H, 1]$. To complete the proof of UAS we must show that for each $\varepsilon>0$ there exists $T>0$ such that $t_{0} \geqslant 0, t>t_{0}+T$, and $\|\phi\|<\delta$ imply $\left|x\left(t ; t_{0}, \phi\right)\right|<\varepsilon$.

To this end, let $\varepsilon>0$ be given and choose $\varepsilon_{1}>0$ with $\varepsilon_{1}<\varepsilon$ and $W_{1}\left(\varepsilon_{1}\right)<W(\varepsilon) / 2$. Now there exists $\varepsilon_{2}>0$ such that $\left\|x_{t}\left(t_{0}, \phi\right)\right\|<\varepsilon_{2}$ implies $W_{2}\left(\left\||| x_{t}\left(t_{0}, \phi\right)\right\| \mid\right)<W(\varepsilon) / 2$. That is,

$$
\begin{aligned}
W_{2}\left(\left\|\left|x_{t}\left(t_{0}, \phi\right)\right|\right\|\right) & =W_{2}\left(\left[\int_{-h}^{0} \sum_{i=1}^{n} x_{i}^{2}(t+s) d s\right]^{1 / 2}\right) \\
& \leqslant W_{2}\left(\left[n h \varepsilon_{2}^{2}\right]^{1 / 2}\right)<W(\varepsilon) / 2
\end{aligned}
$$

if $\left\|x_{t}\left(t_{0}, \phi\right)\right\|<\varepsilon_{2}$ and $\varepsilon_{2}$ is small enough. Let $\varepsilon_{3}=\min \left[\varepsilon_{1}, \varepsilon_{2}\right]$.

With these choices, notice that if a solution satisfies $\left|x\left(t ; t_{0}, \phi\right)\right|<\varepsilon_{3}$ on an interval of length $h$, we then have $\left|x\left(t ; t_{0}, \phi\right)\right|<\varepsilon$ for all future time. This is merely the uniform stability argument once more. For, if $\left|x\left(t ; t_{0}, \phi\right)\right|<\varepsilon_{3}$ for $t_{1}-h \leqslant t \leqslant t_{1}$, then this is equivalent to $\left\|x_{t_{1}}\left(t_{0}, \phi\right)\right\|<\varepsilon_{3} \leqslant \varepsilon_{2}$. Then for $t \geqslant t_{1}$ we have

$$
\begin{aligned}
W\left(\left|x\left(t ; t_{0}, \phi\right)\right|\right) & \leqslant V\left(t, x_{t}\left(t_{0}, \phi\right)\right) \leqslant V\left(t_{1}, x_{t_{1}}\left(t_{0}, \phi\right)\right) \\
& \leqslant W_{1}\left(\left|x\left(t_{1} ; t_{0}, \phi\right)\right|\right)+W_{2}\left(||\left|x_{t_{1}}\left(t_{0}, \phi\right)\right|||\right) \\
& <W_{1}\left(\varepsilon_{1}\right)+W(\varepsilon) / 2<W(\varepsilon) .
\end{aligned}
$$

Now recall that $\phi \in C_{H},\|\phi\|<\delta$, and $t \geqslant t_{0}$ yields $V\left(t, x_{t}\left(t_{0}, \phi\right)\right) \leqslant$ $V\left(t_{0}, \phi\right) \leqslant W_{1}(\delta)+W_{2}\left(\left[n h \delta^{2}\right]^{1 / 2}\right)={ }^{\text {def }} \mu$. Also, $V^{\prime}\left(t, x_{t}\left(t_{0}, \phi\right)\right) \leqslant$ $-W_{3}\left(\left|x\left(t, t_{0}, \phi\right)\right|\right)$ so there exists $T_{1}>h$ such that $\left|x\left(t ; t_{0}, \phi\right)\right| \geqslant \varepsilon_{3}$ must fail for some value of $t$ in each interval of length $T_{1}$. To see this, note that if $\left|x\left(t, t_{0}, \phi\right)\right| \geqslant \varepsilon_{3}$ on an interval $\left[t_{1}, t_{2}\right]$, then

$$
\begin{aligned}
V\left(t_{2}, x_{t_{2}}\left(t_{0}, \phi\right)\right) & \leqslant V\left(t_{1}, x_{t_{1}}\left(t_{0}, \phi\right)\right)-W_{3}\left(\varepsilon_{3}\right)\left(t_{2}-t_{1}\right) \\
& \leqslant \mu-W_{3}\left(\varepsilon_{3}\right)\left(t_{2}-t_{1}\right)<0
\end{aligned}
$$


if $t_{2}-t_{1}>\mu / W_{3}\left(\varepsilon_{3}\right)$, a contradiction to $V \geqslant 0$.

Thus, for each $\phi \in C_{H}$ with $\|\phi\|<\delta$ there is a sequence $\left\{t_{n}\right\}$ monotone to $+\infty$ with $\left|x\left(t_{n} ; t_{0}, \phi\right)\right|<\varepsilon_{3}$. By choosing a subsequence we can say $t_{n}+h<$ $t_{n+1}$. In particular, when $\phi$ and $t_{0}$ are given with $\|\phi\|<\delta$ and $t_{0} \geqslant 0$, we may choose $t_{1} \in\left[t_{0}, t_{0}+T_{1}\right], t_{2} \in\left[t_{0}+2 T_{1}, t_{0}+3 T_{1}\right], t_{3} \in\left[t_{0}+4 T_{1}, t_{0}+\right.$ $\left.5 T_{1}\right], \ldots$ The $t_{i}$ depend on $\phi$, but the indicated interval in which $t_{i}$ lies is independent of $\phi$ whenever $\|\phi\|<\delta$.

Consider now the sequence of functions $\left\{x_{t_{k}}\left(t_{0}, \phi\right)\right\}$ and examine those members satisfying $W_{2}\left(\left\|x_{t_{k}}\left(t_{0}, \phi\right) \mid\right\|\right) \geqslant W(\varepsilon) / 2$. This yields $\left\||| x_{t_{k}}\left(t_{0}, \phi\right) \mid\right\| \geqslant \sqrt{\bar{\alpha}}$, for some $\bar{\alpha}>0$ so that $\sum_{i=1}^{n} \int_{-h}^{0} x_{i}^{2}\left(t_{k}+s\right) d s \geqslant \bar{\alpha}$. From this we obtain

$$
\sum_{i=1}^{n}(1 / n) \int_{t_{k}-h}^{t_{k}} x_{i}^{2}(s) d s \geqslant \bar{\alpha} / n \stackrel{\text { def }}{=} \alpha
$$

where the $x_{i}$ are the components of $x_{t_{k}}\left(t_{0}, \phi\right)$.

We now play the function $W_{3}$ against $W_{2}$. For $t>t_{k}$ we have

$$
\begin{aligned}
V\left(t, x_{t}\left(t_{0}, \phi\right)\right) & \leqslant \mu-\int_{t_{0}}^{t} W_{3}\left(\left|x\left(u ; t_{0}, \phi\right)\right|\right) d u \\
& \leqslant \mu-\sum_{i=2}^{k} \int_{t_{i}-h}^{t_{i}} W_{3}\left(\left|x\left(t ; t_{0}, \phi\right)\right|\right) d t \\
& \leqslant \mu-\sum_{i=2}^{k} \int_{t_{i}-h}^{t_{i}} W_{3}\left([1 / n] \sum_{j=1}^{n} x_{j}^{2}\left(t ; t_{0}, \phi\right)\right) d t
\end{aligned}
$$

as $x_{j}^{2}\left(t ; t_{0}, \phi\right) \leqslant H^{2}<1$.

But from (*) and the lemma we conclude that

$$
\int_{t_{i}-h}^{t_{i}} W_{3}\left([1 / n] \sum_{j=1}^{n} x_{j}^{2}\left(t ; t_{0}, \phi\right)\right)>\beta
$$

for some $\beta>0$ whenever (*) holds. Thus, if (*) holds for $x_{t_{1}}, \ldots, x_{t_{k}}$, then $V\left(t, x_{t}\left(t_{0}, \phi\right)\right) \leqslant \mu-(k-1) \beta$. Now there exists $N$ such that $k>N$ yields $\mu-(k-1) \beta<0$.

As $V \geqslant 0$, we conclude that (*) fails for some $x_{t_{i}}\left(t_{0}, \phi\right)$ for $1 \leqslant i \leqslant N$. Thus, for that $i$, we have $W_{2}\left(\left\|x_{t_{i}}\left(t_{0}, \phi\right)\right\| \|\right)<W(\varepsilon) / 2$ and, consequently, as $\left|x\left(t_{i} ; t_{0}, \phi\right)\right|<\varepsilon_{3}$ we have $\left|x\left(t ; t_{0}, \phi\right)\right|<\varepsilon$ for $t \geqslant t_{i}$.

Finally, we claim that $t_{N}<t_{0}+2 N T_{1}$ so that $T=2 N T_{1}$ suffices. To see this, recall that $\left|x\left(t ; t_{0}, \phi\right)\right| \geqslant \varepsilon_{3}$ fails for some $t$ in every interval of length $T_{1}$ and $T_{1}>h$. This completes the proof.

As a simple example, let

$$
x^{\prime}(t)=-(t+3) x(t)+x(t-1)
$$

and

$$
V\left(t, x_{t}\right)=x^{2}(t) / 2+\int_{t-1}^{t} x^{2}(s) d s
$$


Then

$$
\begin{aligned}
V^{\prime}\left(t, x_{t}\right) & =-(t+3) x^{2}(t)+x(t) x(t-1)+x^{2}(t)-x^{2}(t-1) \\
& <-x^{2}(t)=-W_{3}(|x(t)|)
\end{aligned}
$$

when $W_{3}(r)=r^{2}$. The upper and lower wedges on $V$ are clear. We conclude that the zero solution is UAS.

\section{REFERENCES}

1. T. Yoshizawa, Stability theory by Lyapunov's second method, Math. Soc. Japan, 1966.

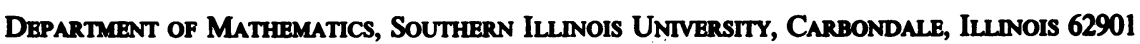

